\title{
Wavelet Analysis of Doppler Flow Velocity Waveforms from the Ocular Circulation Detects Abnormalities in Micro-vascular Function in Heart Failure
}

Kristopher S Lyons ${ }^{1 *}$, Aaron McCann ${ }^{2}$, Auleen Millar ${ }^{1}$, Vivienne McClenaghan ${ }^{1}$, Caroline Bleakley ${ }^{1}$, Gary E McVeigh ${ }^{1}$ and Mark T Harbinson ${ }^{1}$

${ }^{1}$ Centre for Vision and Vascular Science, School of Medicine, Dentistry and Biomedical Sciences, Queens University Belfast, United Kingdom

${ }^{2}$ Regional Medical Physics Service, Belfast Health and Social Care Trust, United Kingdom

\begin{abstract}
Aims: Many pathological processes may result in Heart Failure (HF), however, the final clinical phenotype appears to be common to all aetiologies. Microvascular abnormalities in HF have been demonstrated in a variety of vascular beds however the effect of HF on the retrobulbar circulation is not known. We employed a novel method of analysing blood flow velocity waveforms in order to quantify changes in the Doppler signal obtained from the retrobulbar arteries between HF patients and controls. FMD was also assessed.

Methods and Results: 32 patients with systolic HF on maximal medical therapy and 10 age and sex-matched controls were studied. Doppler waveforms were obtained from the ophthalmic, central retinal and brachial artery in all subjects. Waveforms were analysed using a wavelet technique which expressed each waveform in constituent frequency bands, allowing direct comparisons to be made. Significant differences were found in ophthalmic and central retinal waveforms but not brachial waveforms between patients and controls. Flow mediated dilatation was also found to be abnormal in patients.
\end{abstract}

Conclusion: This study suggests that abnormalities in microvascular function in the retrobulbar circulation are present in HF and that these changes can be detected by a novel wavelet analysis technique.

Keywords: Heart failure; Endothelial dysfunction; Ultrasound

\section{Introduction}

Many pathological processes may result in heart failure (HF), however, the final clinical phenotype appears to be common to all aetiologies. Activation of neurohormonal systems and cardiac remodelling occur in a predictable sequence [1]. As well as the biochemical abnormalities, microvascular dysfunction also occurs and has been demonstrated in a variety of vascular beds [2-4]. Methods used to detect microvascular abnormalities in HF however, have produced conflicting results and some traditional methods such as flow mediated dilatation (FMD) are limited by technical issues, an unclear relationship to clinical events and a possible oversimplification of the complex processes involved in production of the vasodilator response [5].

Our department has developed a novel method of analysing Doppler flow velocity waveforms obtained from vascular sites of interest which can detect differences in waveforms between controls and a variety of disease models associated with endothelial dysfunction [610]. Blood flow velocity waveforms are composed of a combination of a forward wave due to left ventricular stroke volume and reflected waves from the microcirculation. When the cardiac output and therefore the forward flow remain the same, changes in the wave shape must represent changes in reflected waves, suggesting changes in number and diameter of recruited vessels in the distal microcirculation [11]. Wavelet analysis is an efficient method of analysing these signals. The waveform is compared with a selected mathematical function known as a wavelet which allows frequency correlations to be made at individual points along the entirety of the signal maintaining information about frequency in the time domain [10]. All data points are used therefore providing a more thorough analysis than traditional time domain techniques such as resistive index ([RI] defined as peak systolic velocity - end diastolic velocity/peak systolic velocity) and pulsatility index ([PI] defined as peak systolic velocity - end diastolic velocity/mean velocity) which only utilise a small number of data points from the wave form. Accessing the retrobulbar circulation allows interrogation of arteries in close proximity to the microcirculatory bed and so the effect of changes in the microcirculation should be more easily detectable. This method may therefore allow temporal tracking of changes in the microcirculation in individuals using a simple practical method and potentially allow detection of microvascular dysfunction at an early stage in diseases associated with endothelial dysfunction.

Microvascular abnormalities in HF have been demonstrated in several locations however the effect of $\mathrm{HF}$ on the retrobulbar circulation is not known. In this study we employed wavelet analysis to quantify changes in Doppler flow velocity waveforms obtained from the ophthalmic, central retinal and brachial arteries between HF patients and a group of age and sex matched controls. FMD was also assessed.

\section{Materials and Methods}

\section{Patient population}

32 patients with HF due to systolic dysfunction and ejection fraction $<45 \%$ were recruited from specialist HF clinics within the Belfast Health and Social Care Trust. Patients were receiving maximal tolerated HF therapy with no admissions for exacerbations in the previous 6 months.

*Corresponding author: Kristopher S Lyons, 47 Beech Green, Doagh Co Antrim, BT39 0QB, United Kingdom, Tel: +44 7979898493; E-mail: kristopherlyons@ hotmail.co.uk

Received June 29, 2015; Accepted July 17, 2015; Published July 20, 2015

Citation: Lyons KS, McCann A, Millar A, McClenaghan V, Bleakley C (2015) Wavelet Analysis of Doppler Flow Velocity Waveforms from the Ocular Circulation Detects Abnormalities in Micro-vascular Function in Heart Failure. J Cardiovasc Dis Diagn 3: 210. doi:10.4172/2329-9517.1000210

Copyright: ( 2015 Lyons KS, et al. This is an open-access article distributed under the terms of the Creative Commons Attribution License, which permits unrestricted use, distribution, and reproduction in any medium, provided the original author and source are credited. 
Citation: Lyons KS, McCann A, Millar A, McClenaghan V, Bleakley C (2015) Wavelet Analysis of Doppler Flow Velocity Waveforms from the Ocular Circulation Detects Abnormalities in Micro-vascular Function in Heart Failure. J Cardiovasc Dis Diagn 3: 210. doi:10.4172/23299517.1000210

Page 2 of 6

No changes to baseline medication were permitted during the study period. 10 age and sex-matched controls were recruited from within the department. Controls had no history of overt cardiovascular disease however a history of hypertension was permitted due to the age range studied. Written informed consent was obtained from all subjects. The investigation conforms with the principles outlined in the Declaration of Helsinki (2000) and was approved by the local office for research ethics committees in Northern Ireland (ORECNI).

All procedures were carried out in the morning in a temperature controlled laboratory. Participants were requested to fast for 12 hours prior to study procedures. The same investigator performed all ultrasound procedures. Flow waveforms from the central retinal, ophthalmic and brachial arteries were analysed. These sites are easily accessed and brachial artery waveforms have long been studied in relation to endothelial dysfunction using flow mediated dilatation. The retrobulbar vessels allow analysis of arterial waveforms in much closer physical proximity to the capillary bed, thereby in theory maximising the impact of reflected waves on the overall flow velocity waveform.

\section{Ultrasound}

Retrobulbar circulation: The right eye was examined in all cases. Colour Doppler ultrasonography was carried out using a MyLab 70 ultrasound scanner (Esoate, Genoa, Italy) with a $12.5 \mathrm{MHz}$ linear array probe.

Subject positioning and location of vessels was standardized for all subjects. Patients were positioned supine with the head supported on a single pillow. The patients were asked to fix their gaze on a marked point on the ceiling by keeping the left eye open. The ultrasound probe was placed lightly on the closed eyelid with a layer of ultrasound gel and the examiner's hand rested lightly on the forehead to allow adjustment of pressure with the ultrasound transducer. A continuous ECG trace was recorded for every patient. Default vascular imaging settings were selected and grey scale image quality was optimized by adjusting the gain settings. The ophthalmic artery was imaged first using colour Doppler to locate the vessel medial to the optic nerve. The central retinal artery was then located at the posterior aspect of the globe in line with the optic nerve. For both vessels a continuous pulsed Doppler waveform was recorded for approximately 15 cardiac cycles and the complete waveform was stored on the Mylab hard-drive as single screen shots.

Brachial artery: Subject positioning was as for ocular ultrasound. The subject's left arm rested at $90 \mathrm{o}$ on a padded support table and the hand was secured to reduce movement. The brachial artery was imaged in the medial aspect of the upper arm and vascular image settings were optimized. The ultrasound probe was then fixed in position in a clamp to reduce movement artefact. A continuous pulsed Doppler of baseline brachial artery flow was recorded.

\section{Image analysis}

Waveforms were stored as image files and separate files were joined using a specifically designed Doppler Analyser v1.6 software package (Esaote, Genoa, Italy) which produces a text file containing mean velocities at each sample point. The angle of isonation of the ultrasound beam, and unit type $(\mathrm{Hz})$ were extracted from the header.

\section{Signal analysis}

Methods and reproducibility have previously been reported [8-10]. Briefly, signals were passed through a low-pass filter to remove signal content above $30 \mathrm{~Hz}$, then segmented into pulses with removal of poor quality segments. Pulses which differed significantly from others were excluded using an automated error checking system which iteratively compares each pulse with an average pulse template based on the entire signal and then excludes pulses which exceeded a fixed threshold for maximum excursion, minimum excursion and similarity index.

The following time-domain parameters were then calculated for each valid pulse in the signal train:

- $\quad$ Resistive index

- Pulsatility index

- Mean velocity

\section{Wavelet analysis}

A variety of wavelets are available and an appropriate wavelet is selected based on the type of signal being analysed. Our group used a pre-specified wavelet for analysis which had previously been chosen from a variety of wavelets based on its ability to determine the frequency content of a synthesised blood velocity waveform. From the 6 wavelets tested, the discrete Meyer (DMey) wavelet performed best for analysis of time stationary and non-time stationary waveforms [9].

The frequency content was divided into 9 arbitrary frequency bands from 0 to $30 \mathrm{~Hz}$, to allow comparison of waveforms using standard comparitors. There is no current data to suggest that the frequency bands have any particular physiological significance.

\section{Flow mediated dilatation}

Subject positioning was as for brachial artery ultrasound. The Mylab visual display was linked to a personal computer with a NI PCI-1407 peripheral component interconnect (PCI) video capture card (National Instruments, Texas, USA). The picture was analysed in real-time, using customised software (On-line Vascular Image Analysis v.9.61, London, UK) developed on LabVIEW (National Instruments, Texas, USA) as used in similar work within the department. A region of the brachial artery where the vessel wall was clearly seen was selected with a manually drawn window and the software package automatically tracked the vessel walls making measurements of diameter at a rate of $25 \mathrm{~Hz}$. Vessel diameter was measured from before cuff inflation until 2 minutes following cuff deflation.

\section{Results}

32 patients were recruited in total. The majority were male (78\%). Mean age was 63.5 years (SD 11). Mean BMI was $30.4 \mathrm{~kg} / \mathrm{m}^{2}$ (SD 6.0). 7 patients had a BMI $>35 \mathrm{~kg} / \mathrm{m}^{2} .6$ patients $(20 \%)$ were diabetic and $12(37.5 \%)$ had a history of atrial fibrillation. 4 (12.5\%) patients had significant valvular heart disease (classified as at least moderate valve regurgitation or stenosis) and $7(21.9 \%)$ had a diagnosis of COPD, receiving inhaled therapy. 6 patients $(18.8 \%)$ were current smokers and 15 (46.9\%) were ex-smokers. 14 patients $(43.8 \%)$ were taking antiplatelet therapy (all aspirin) and 14 (43.8\%) were taking warfarin. Patients were on typical HF therapy to include beta blockers (90.6\%), ACE inhibitors or ARBs (96.9\%) and spironolactone (56.3\%). 16 patients (50\%) had non-ischaemic cardiomyopathy. All patients were in NHYA class II (46.9\%) or III (53.1\%) and mean EF was 31\% (SD 6.9). 8 patients (25\%) had an implantable cardiac device.

10 age and sex matched control subjects were recruited. Baseline demographics are shown in Table 1. BMI was higher in the patient group. Due to the age of controls required, a history of hypertension was allowed however no controls had any history of overt cardiovascular 
Citation: Lyons KS, McCann A, Millar A, McClenaghan V, Bleakley C (2015) Wavelet Analysis of Doppler Flow Velocity Waveforms from the Ocular Circulation Detects Abnormalities in Micro-vascular Function in Heart Failure. J Cardiovasc Dis Diagn 3: 210. doi:10.4172/23299517.1000210

Page 3 of 6

disease. 2 control subjects had a history of hypertension.

\section{FMD}

FMD was significantly greater in the control group compared to patients (5.9\% [IQ range $3.9-12.9$ ] vrs $3.8 \%$ [IQ range $0.2-6.2$ ] $\mathrm{p}=0.024$ ) (Figure 1). This difference was apparent in patients with HF of ischaemic $(\mathrm{p}=0.043)$ and non-ischaemic $(\mathrm{p}=0.048)$ aetiologies.

\section{Opthalmic artery waveforms}

There were no significant differences in the time domain parameters RI and PI, however, mean amplitude of frequency bands 5 and 8 obtained by wavelet analysis was significantly higher in patients compared to controls (Table 2 and Figure 2).

When ischaemic and non-ischaemic aetiologies were considered seperately, differences remained significant for both bands in patients with ischaemic cardiomyopathy $(\mathrm{p}=0.043$ and $\mathrm{p}=0.002$ for 5 and 8 respectively) and for band 8 but not band 5 in patients with non-ischaemic cardiomyopathy $(\mathrm{p}=0.136$ and $\mathrm{p}=0.020$ for 5 and 8 respecitively).

\section{Central retinal artery waveforms}

There were no significant differences in time domain parameters, however, as for opthalmic artery waveforms, mean amplitude of frequency bands 5 and 8 obtained by wavelet analysis were significantly higher in patients compared to controls (Table 3 and Figure 3).

When ischaemic and non-ischaemic aetiologies were considered

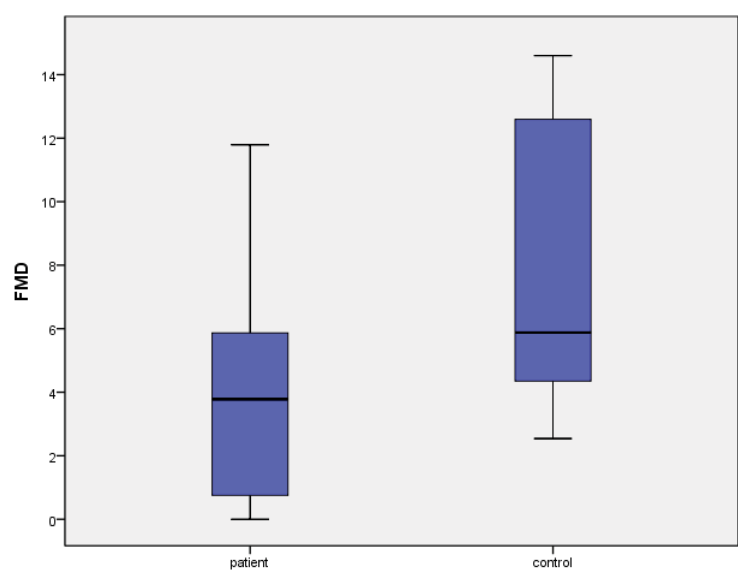

Figure 1: Boxplots showing median and interquartile range for FMD (flowmediated dilatation) in patients and controls.

\begin{tabular}{|c|c|c|c|c|c|c|c|}
\hline & & 1. & Patient & 2. & Control & $\begin{array}{l}3 . \\
\text { value }\end{array}$ & p \\
\hline 4. & Age & $\begin{array}{l}5 . \\
(1.94)\end{array}$ & 6 & $\begin{array}{l}6 . \\
(4.13)\end{array}$ & 6 & 7. & 0.92 \\
\hline $\begin{array}{l}8 . \\
9 . \\
10 .\end{array}$ & $\begin{array}{l}\text { Sex } \\
\text { male } \\
\text { female }\end{array}$ & $\begin{array}{l}11 . \\
12 . \\
13 .\end{array}$ & $\begin{array}{l}25 \\
7\end{array}$ & $\begin{array}{l}14 . \\
15 . \\
16 .\end{array}$ & $\begin{array}{l}7 \\
3\end{array}$ & $\begin{array}{l}17 . \\
18 .\end{array}$ & 0.68 \\
\hline 19. & BMI & $\begin{array}{l}20 . \\
(1.06)\end{array}$ & 30.4 & $\begin{array}{l}21 . \\
(1.29)\end{array}$ & $25 \cdot 3$ & 22. & .007 \\
\hline 23. & Smoker & 24. & 6 & 25. & 1 & 26. & 1.00 \\
\hline
\end{tabular}

Table 1: Baseline demographics of patients and controls.

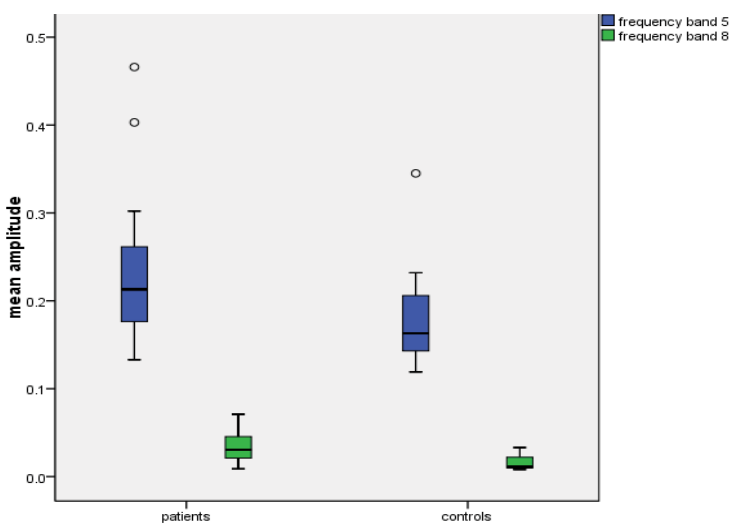

Figure 2: Mean amplitude of frequency bands 5 and 8 for patients and controls for opthalmic waveforms.

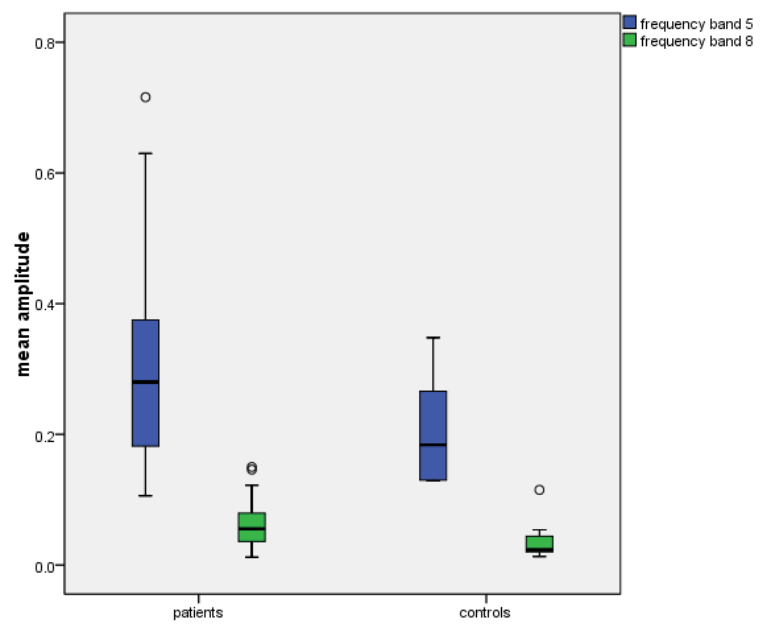

Figure 3: Mean amplitude of frequency bands 5 and 8 in patients and controls for central retinal artery waveforms.

seperately, differences remained significant for both bands in patients with ischaemic cardiomyopathy $(\mathrm{p}=0.009$ and $\mathrm{p}=0.007$ for 5 and 8 respectively) but not in patients with non-ischaemic cardiomyopathy $(\mathrm{p}=0.182$ and $\mathrm{p}=0.220$ for 5 and 8 respecitively).

\section{Brachial artery waveforms}

There was no difference between time domain parameters between patients and controls. There was also no difference between frequency bands derived by wavelet analysis (Table 4).

\section{Discussion}

In this comparison of patients with systolic HF and age and sex matched controls, FMD was found to be significantly lower in HF patients. Differences were also apparent in the mean amplitude of 2 frequency bands obtained by wavelet analysis in opthalmic and central retinal artery waveforms. Similar changes were seen for both vessels, with lower mean amplitude of freqency bands 5 and 8 in controls compared to HF patients. Examples of opthalmic and central retinal waveforms for patients and controls are shown in Figure 4 ( $a$ and b). 
Citation: Lyons KS, McCann A, Millar A, McClenaghan V, Bleakley C (2015) Wavelet Analysis of Doppler Flow Velocity Waveforms from the Ocular Circulation Detects Abnormalities in Micro-vascular Function in Heart Failure. J Cardiovasc Dis Diagn 3: 210. doi:10.4172/23299517.1000210

Page 4 of 6

\begin{tabular}{|c|c|c|c|}
\hline & Patients & Controls & P \\
\hline RI & $0.790(0.724-0.819)$ & $0.789(0.670-0.891)$ & 0.738 \\
\hline PI & $1.704(1.338-2.006)$ & $1.617(1.106-2.086)$ & 0.782 \\
\hline DWT & & & 0.255 \\
\hline Band 1 & $0.010(0.009-0.013)$ & $0.007(0.006-0.012)$ & 0.092 \\
\hline Band 2 & $0.031(0.024-0.036)$ & $0.023(0.019-0.035)$ & 0.359 \\
\hline Band 4 & $0.065(0.055-0.086)$ & $0.060(0.049-0.082)$ & 0.255 \\
\hline Band 5 & $0.122(0.089-0.147)$ & $0.107(0.070-0.133)$ & \\
\hline Band 6 & $0.213(0.176-0.263)$ & $0.163(0.136-0.219)$ & \\
\hline Band 7 & $0.317(0.278-0.352)$ & $0.256(0.236-0.405)$ & \\
\hline Band 9 & $0.051(0.027-0.097)$ & $0.057(0.037-0.099)$ & \\
\hline Band & $0.030(0.021-0.046)$ & $0.011(0.010-0.025)$ & 0.525 \\
\hline
\end{tabular}

Table 2: RI (resistive index), PI (pulsatility index) and mean amplitude of wavelet frequency bands for patients and controls for opthalmic artery waveforms.

\begin{tabular}{|c|c|c|c|}
\hline & Patients & Controls & P \\
\hline RI & $1.01(1.00-1.02)$ & $1.01(0.95-1.01)$ & 0.286 \\
\hline PI & $2.43(1.78-3.04)$ & $2.21(1.49-2.59)$ & 0.260 \\
\hline DWT & & & \\
\hline Band 1 & $0.037(0.028-0.045)$ & $0.030(0.019-0.040)$ & 0.163 \\
\hline Band 2 & $0.075(0.066-0.098)$ & $0.064(0.044-0.083)$ & 0.154 \\
\hline Band 3 & $0.115(0.085-0.140)$ & $0.088(0.049-0.133)$ & 0.202 \\
\hline Band 5 & $0.166(0.108-0.217)$ & $0.108(0.076-0.161)$ & 0.069 \\
\hline Band 6 & $0.280(0.181-0.376)$ & $0.184(0.130-0.283)$ & \\
\hline Band 7 & $0.529(0.257-0.753)$ & $0.347(0.245-0.610)$ & \\
\hline Band 8 & $0.087(0.052-0.152)$ & $0.080(0.056-0.113)$ & \\
\hline Band 9 & $0.056(0.036-0.080)$ & $0.024(0.019-0.047)$ & \\
\hline
\end{tabular}

Table 3: RI (resistive index), PI (pulsatility index) and mean amplitude of wavelet frequency bands in patients and controls for central retinal artery waveforms.

\begin{tabular}{|c|c|c|}
\hline & Patients & \multicolumn{1}{c|}{ Controls } \\
\hline RI & $1.21(0.99-1.29)$ & $1.21(1.04-1.28)$ \\
\hline PI & $6.36(3.07-8.73)$ & $8.74(5.10-12.29)$ \\
\hline DWT & & \\
\hline Band 1 & $0.034(0.018-0.051)$ & 0.699 \\
\hline Band 2 & $0.077(0.037-0.103)$ & $0.032(0.025-0.063)$ \\
\hline Band 3 & $0.174(0.099-0.276)$ & $0.079(0.061-0.145)$ \\
\hline Band 4 & $0.474(0.305-0.674)$ & $0.241(0.160-0.360)$ \\
\hline Band 5 & $0.828(0.440-1.067)$ & $0.553(0.421-0.865)$ \\
\hline Band 6 & $0.0 .880(0.467-1.119)$ & $0.873(0.747-1.281)$ \\
\hline Band 7 & $0.133(0.053-0.242)$ & $0.893(0.842-1.311)$ \\
\hline Band 9 & $0.0 .052(0.033-0.083)$ & $0.266(0.098-0.450)$ \\
\hline
\end{tabular}

Table 4: RI (resistive index), PI (pulsatility index) and mean amplitude of wavelet frequency bands in patients and controls for brachial artery waveforms.

However when brachial artery waveforms were similarly analysed, no differences were found. This may be explained by the proximity of the artery in question to the terminal vascular bed. Opthalmic and central retinal arteries are located in close proximity to the retinal circulation. Therefore wave reflection from the microvascular bed will have a significant effect on the overall morphology of the Doppler waveform. The brachial artery however, is further from the terminal vascular bed in the hand, and therefore it could be argued that the effect of reflected waves would be greatly diminished. This can be seen in the morphology of the waveforms. Those obtained from the brachial artery are realtively 'featureless' compared to those obtained from retrobulbar vessels which have a greater complexity with changes evident in the diastolic decay even on visual examination. Example waveforms are shown in Figure 4c.
It therefore follows that to detect changes in Doppler waveforms due to changes in microvascular function, arteries must be examined in close proximity to the terminal vascular bed. Other work in our department has prod uced similar results in patients with diabetes. Differences in retrobulbar waveforms have been demonstrated between controls and subjects with diabetes, however analysis of baseline carotid artery waveforms between both groups showed no difference [10].

When ischaemic and non-ischaemic groups were analysed seperately, FMD was found to be reduced compared to controls in both groups, however differences were less apparent for opthalmic waveforms between controls and the non-ischaemic group, while no difference was found for central retinal waveforms between controls and the non-ischaemic group. Endothelial dysfunction as assessed by FMD 
Citation: Lyons KS, McCann A, Millar A, McClenaghan V, Bleakley C (2015) Wavelet Analysis of Doppler Flow Velocity Waveforms from the Ocular Circulation Detects Abnormalities in Micro-vascular Function in Heart Failure. J Cardiovasc Dis Diagn 3: 210. doi:10.4172/23299517.1000210

Page 5 of 6
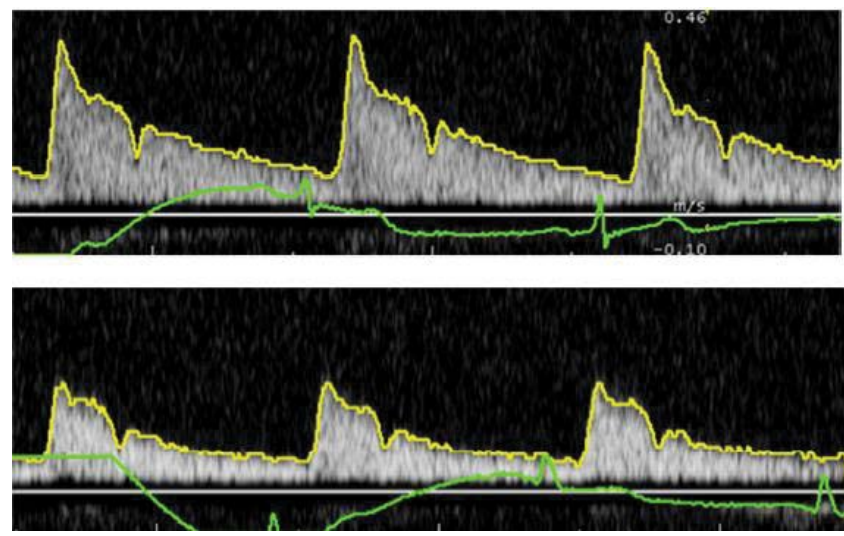

Figure 4a: Top image shows opthalmic waveform obtained from healthy control. Bottom image from patient. Differences are apparent in the systolic peak and gradient of the diastolic decay.
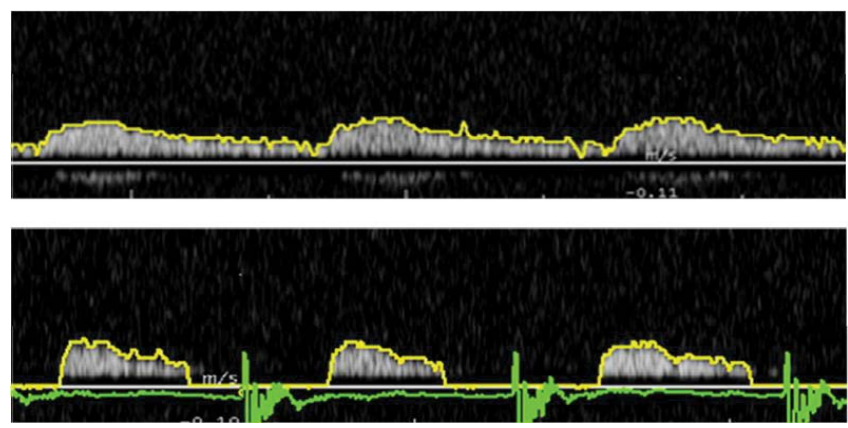

Figure 4b: Top image shows central retinal waveform obtained from healthy control. Bottom image from patient. Differences are apparent in duration of flow and diastolic decay.
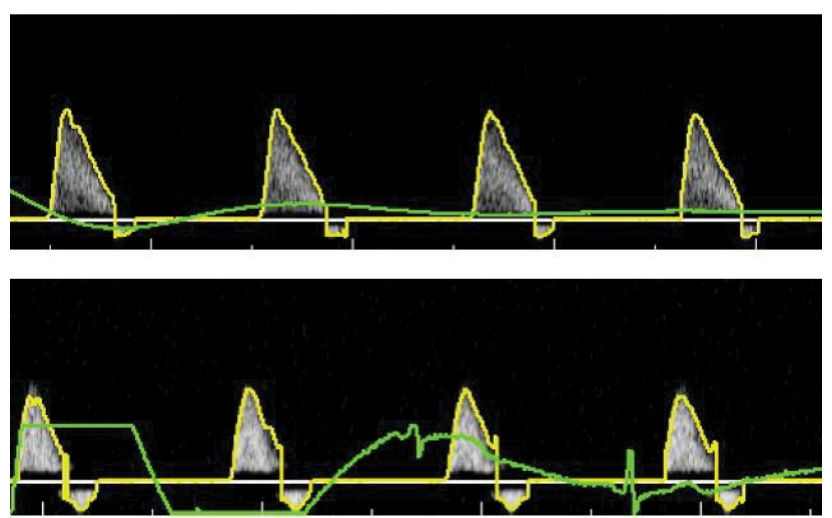

Figure 4c: Top image shows brachial artery waveform from healthy control. Bottom image from patient. Little difference is apparent visually between waveforms.

has been previously demonstrated in patients with ischaemic and nonischaemic cardiomyopathy, a finding confirmed in this study [12,13]. However FMD is traditionally recognised as a measure of the release of vasoactive substances, particularly nitric oxide (NO) in response to shear stress, and NO bioavailability is affected by many factors which occur as a result of the heart failure syndrome such as increased free radical production [14]. The blood flow velocity waveforms represent a forward wave due to the cardiac output and a reverse wave reflected from the microvasculature. It may therefore be that there are structural changes within the microvasculature of patients with ischaemic cardiomyopathy resulting in changes to the waveform morphology, that are not apparent in those with non-ischaemic aetiology. Ofcourse, the small number of patients in the subgroups mean that any differences between these groups should be interpreted with caution.

This study therefore suggests that abnormalities in microvascular function in the retrobulbar circulation are present in HF. Endothelial dysfunction is a systemic manifestation of the neurohormonal abnormalities present in HF. Investigation of various microvascular beds using a variety of methods has demonstrated differences between HF patients and controls. De Backer et al. demonstrated abnormalities in the sublingual circulation with reduced perfusion of small vessels in HF patients while several authors have shown that skin blood flow is also abnormal $[2,3]$. To study the microcirculation, it must be possible to in some way interrogate the area of interest. The eye represents a desirable location as the retrobulbar vessels are easily assessed non-invasively using ultrasound. No intravenous access or pharmacotherapy are required, and the central retinal and opthalmic arteries provide easily identifiable targets for repeated measures in single subjects as well as comparisons between subjects, as opposed to using the skin model where random areas are chosen, and significant heterogeneity may exist between different regions. There is also strong correlation between the presence of retrobulbar vascular disease and the occurrence of heart failure, from the diabetic population. Diabetic patients with retinopathy have a much higher incidence of heart failure compared to those without [15].

Microvasular dysfunction in HF can be demonstrated in a variety of vascular beds, and this study has shown that changes in Doppler waveforms obtained from the retrobulbar circulation are detectable between patients with $\mathrm{HF}$ and age and sex matched controls. Differences in the traditional methods used to assess flow waveforms (PI and RI) were not apparent, suggesting that the wavelet analysis which analyses all data points in the waveform is a more sensitive method of detecting subtle changes in waveform morphology.

\section{Acknowledgment of Support}

This research was supported by a Doctoral Fellowship Award from the Research and Development office of the Health and Social Care Public Health Agency.

\section{References}

1. Francis GS (2001) Pathophysiology of chronic heart failure. Am J Med 110(supplement I): 37-46.

2. Green DJ, Maiorana AJ, Siong JH, Burke V, Erickson M, et al. (2006) Impaired skin blood flow response to environmental heating in chronic heart failure. Eur Heart J 27: 338-343.

3. De Backer D, Creteur J, Dubois MJ, Sakr Y, Vincent JL (2004) Microvascular alterations in patients with acute severe heart failure and cardiogenic shock. Am Heart J 147: 91-99.

4. Balmain S, Padmanabhan N, Ferrell WR, Morton JJ, McMurray JJV (2007) Differences in arterial compliance, microvascular function and venous capacitance between patients with heart failure and either preserved or reduced left ventricular systolic function. Eur J Heart Fail 9: 865-871.

5. Hamilton P, Lockhart CJ, McCann AJ, Agnew CE, Harbinson MT, et al. (2011) Flow-mediated dilatation of the brachial artery is a poorly reproducible indicator of microvascular function in Type I diabetes mellitus. QJM 104: 589-597.

6. Wright SA, O'Prey FM, Hamilton PK, Lockhart CJ, McCann A, et al. (2009) Colour doppler ultrasound of the ocular circulation in patients with systemic lupus erythematosus identifies altered microcirculatory haemodynamics. Lupus 18: 950-957. 
Citation: Lyons KS, McCann A, Millar A, McClenaghan V, Bleakley C (2015) Wavelet Analysis of Doppler Flow Velocity Waveforms from the Ocular Circulation Detects Abnormalities in Micro-vascular Function in Heart Failure. J Cardiovasc Dis Diagn 3: 210. doi:10.4172/23299517.1000210

Page 6 of 6

7. Agnew CE, McCann AJ, Lockhart CJ, Hamilton PK, McVeigh GE, et al. (2011) Comparison of RootMUSIC and discrete wavelet transform analysis of doppler ultrasound blood flow waveforms to detect microvascular abnormalities in type I diabetes. Biomed Eng 58: 861-867.

8. Lockhart CJ, McCann A, Agnew CA, Hamilton PK, Quinn CE, et al. (2011) Impaired microvascular properties in uncomplicated type 1 diabetes identified by Doppler ultrasound of the ocular circulation. Diabetes Vasc Dis Re 8: 211220.

9. Quinn CE, Hamilton PK, McCann AJ, Agnew CE, Millar AM, et al. (2011) Ocular blood flow analysis detects microvascular abnormalities in impaired glucose tolerance. Microcirculation 18: 532-540.

10. Hamilton PK, McCann AJ, Agnew CE, Millar A, McClenaghan VO, et al. Detecting early microvascular disease in type 1 diabetes: wavelet transform analysis of Doppler blood velocity waveforms. Brit J Diabetes Vasc Dis 12: 40-47.

11. Lockhart CJ, Hamilton PK, Quinn CE, McVeigh GE (2009) End-organ dysfunction and cardiovascular outcomes: the role of the microcirculation. Clin Sci (Lond) 116: 175-190.

12. Marti CN, Gheorghiade M, Kalogeropoulos AP, Georgiopoulou VV, Quyyum AA, et al. (2012) Endothelial dysfunction, arterial stiffness, and heart failure. $J$ Am Coll Cardiol 60: 1455-1469.

13. Klosinska M, Rudzinski T, Grzelak P, Stefanczyk L, Drozdz J, et al. (2009) Endothelium-dependent and -independent vasodilation is more attenuated in ischaemic than in non-ischaemic heart failure. Eur $\mathrm{J}$ Heart Fail 11: 765-770.

14. Münzel T, Harrison DG (1999) Increased superoxide in heart failure: a biochemical baroreflex gone awry. Circulation 100: 216-218.

15. Wong TY, Rosamond W, Chang PP, Couper DJ, Sharrett AR, et al. (2005) Retinopathy and risk of congestive heart failure. JAMA 293: 63-69. 